\title{
FURTHER NOTES ON THE EVOLUTION OF DOMINANCE
}

\author{
W. J. EWENS \\ Australion National University
}

Received 23.ii. 65

\section{INTRODUCTION}

WHETHER one accepts or rejects Fisher's theory (Fisher, I928a, $b$, I929) of the evolution of dominance, it is interesting to note that Fisher originally developed it using an incorrect formula and that had he been aware of the correct formula he would not have advanced his theory at all. Fisher's formula has been discussed recently by the author (Ewens, I965) and is discussed further in this paper. Also, an analysis is made of a process which Parsons and Bodmer (I96I) suggest may lead to the evolution of dominance, various factors concerned in the evolution of dominance process are summarised and their relative importance discussed.

\section{FISHER'S ANALYSIS}

We suppose that we are primarily interested in a locus " $A$ " at which alleles $A$ and $a$ can occur. $A$ is supposed to have sufficient selective advantage over $a$ so that the occurrence of $a$ comes about only by mutation (at rate $u$ ) from $A$ to $a$. The allele $a$ will then appear almost always in heterozygotes. Imagine now a modifying locus " $M$ ", with alleles $M$ and $m$, for which the allele $M$ modifies the heterozygote $A a$ towards the homozygote $A A$. We may suppose that such modification may depend on whether $M$ itself occurs in homozygotes or heterozygotes. Specifically, it is assumed that the selective advantages of the various types are given by the following array:

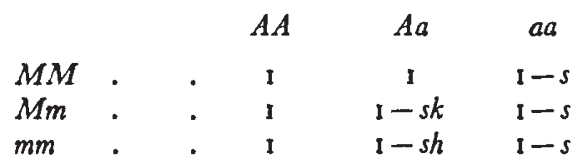

Fisher was interested in the case $k=0$, which we now consider in some detail. If initially the allele $M$ has no effect on the " $A$ " locus, and all heterozygotes $A a$ have the same selective advantage I-sh, then the equilibrium frequency of $A$ is $1-u / h s$. If the allele $M$ now begins to modify $A a$, and the selective advantages then change to those shown above, then the initial increase $\Delta q_{M}$ in the frequency of $M$ was shown (Ewens, I 965 ) to be given approximately by

$$
\Delta q_{M}=2 u q_{M}\left(\mathrm{I}-q_{M}\right)^{2} .
$$

The incorrect formula given by Fisher $(1928 a, b, 1929)$ is

$$
\Delta q_{M}=2 u q_{M},
$$


an expression which Fisher apparently uses throughout the whole dominance-modification process. In deriving equation (2), Fisher used a univariate analysis (of the frequency $q_{M}$ ), whereas as has been shown by Moran ( 1964 ), in cases such as the above when selective advantages depend jointly on two loci, a univariate analysis is inadequate and it is necessary to use a bivariate analysis. Specifically, Moran showed that it is not sufficient to consider only the gene frequencies $q_{A}$ and $q_{M}$; rather it is necessary to consider the frequencies $c_{1}, c_{2}, c_{3}$ and $c_{4}$ of the gametes $A M, a M, A m$ and $a m$ immediately before the formation of the zygotes of each generation. In fact the correct formula for $\Delta q_{M}$ was shown in (Ewens, 1965) to be

$$
\Delta q_{M}=2 s h q_{M} c_{3} c_{4}+\mathrm{O}\left(u^{2}\right) \text {. }
$$

If we use the approximations

$$
\begin{aligned}
& c_{3}=\left(\mathrm{I}-q_{M}\right)+\mathrm{O}(u), \\
& c_{4}=u\left(\mathrm{I}-q_{M}\right) / h s+\mathrm{O}\left(u^{2}\right),
\end{aligned}
$$

we derive ( 1 ) immediately from (3). If we use more generally the approximations

then (3) becomes

$$
\begin{aligned}
& c_{3}=q_{A}\left(\mathrm{I}-q_{M}\right), \\
& c_{4}=\left(\mathrm{I}-q_{A}\right)\left(\mathrm{I}-q_{M}\right),
\end{aligned}
$$

$$
\Delta q_{M}=2 s h q_{A}\left(\mathrm{I}-q_{A}\right) q_{M}\left(\mathrm{I}-q_{M}\right)^{2} .
$$

Moran (1964) has shown that the approximation (5) is sufficiently accurate, at least when $s$ is small. The accuracy of the approximation in the present context will be considered later.

Now as the process continues, not only will the frequency $q_{M}$ change, but the frequency $q_{A}$ will also change. In fact, $q_{A}$ will steadily decrease from its initial value $\mathrm{I}-u / h$ s to the equilibrium value of $A$ corresponding to $q_{M}=\mathrm{I}$, namely $\mathrm{I}-\sqrt{u / s}$. Correspondingly, $\Delta q_{M}$ will not always assume the form ( $\mathrm{I})$. In order to derive the general formula for $\Delta q_{M}$ we must proceed as follows. It is easily shown, using the approximations (4) and Moran's (1964) analysis, that

$$
\Delta q_{A}=s q_{A}\left(\mathrm{I}-q_{A}\right)\left[\mathrm{I}-q_{A}+h\left(\mathrm{I}-q_{M}\right)^{2}\left(2 q_{A}-\mathrm{I}\right)\right]-u q_{A} .
$$

The last term on the right-hand side corresponds to the mutation from $A$ to $a$. Passing to continuous time (5) and (6) give jointly

$$
\frac{d q_{A}}{d q_{M}}=\frac{\mathrm{I}-q_{A}+h\left(\mathrm{I}-q_{M}\right)^{2}\left(2 q_{A}-\mathrm{I}\right)}{2 h q_{M}\left(\mathrm{I}-q_{M}\right)^{2}}-\frac{u}{2 \operatorname{sh}\left(\mathrm{I}-q_{A}\right) q_{. M}\left(\mathrm{I}-q_{M}\right)^{2}},
$$

an equation which for all practical purposes should yield correct results in the discrete time case. In order to get the exact expression for $\Delta q_{M}$ we should now solve (7) for $q_{A}$ in terms of $q_{M}$ and substitute the value so obtained in (5). Unfortunately, exact solution of (7) 
does not appear to be simple, and in order to get some idea of the values obtained by $\Delta q_{M}$ we abandon the attempt to find the exact formula and consider rather the following numerical example.

Suppose that $s=0.0 \mathrm{I}, u=10^{-6}, h=\mathrm{I}$, so that initially $q_{A}=1-10^{-4}$. The frequency $q_{A}$ will decrease steadily to the value given by the formula $q_{A}=\mathrm{I}-\sqrt{u / s}$, namely $q_{A}=\mathrm{I}-\mathrm{IO}^{-2}$. Thus during the entire history of the process, $\mathrm{I}-q_{A}$ is always bounded above by $10^{-2}$ so that $\Delta q_{M}$ is always bounded above by

$$
2 \times 10^{-4} \times q_{M}\left(1-q_{M}\right)^{2} .
$$

The quantity of main interest to Fisher (1929) and Wright (1929a, $b$ ) is that function $i$ satisfying

$$
\Delta q_{M}=q_{M}\left(\mathrm{r}-q_{M}\right) i .
$$

On Fisher's incorrect formula, $i$ increases without limit as $q_{M} \rightarrow 1$, a fact which " is an essential point " (Fisher, 1929) of Fisher's theory. The above analysis, however, shows that $i$ is always bounded above by the quantity $2 \times 10^{-4}\left(1-q_{M}\right)$, which is not only small but decreases steadily to zero as $q_{M}$ approaches unity.

Ewens (1965) claimed that Fisher's incorrect formula was a result of failing to use the correct (i.e. Moran's) analysis. However, this is not perhaps too important; his derivation is incorrect for a second, more subtle, reason. Having obtained (essentially) the expression (5), he evaluated $q_{A}$ in terms of $q_{M}$ not by solving equation (7), but by using for $q_{A}$ that value which solves

$$
\Delta q_{A}=\mathrm{o} .
$$

This is not allowable, since the frequency of $A$ is not stationary but is steadily decreasing. Equation (8) will only hold when the equilibrium point $q_{M}=\mathrm{I}$ has been reached. If, following Fisher, we use (6) to solve equation (8), we get (ignoring terms in $\left(1-q_{A}\right)^{2}$ )

$$
\mathrm{I}-q_{A}=\frac{\mathrm{u}}{h s\left(\mathrm{I}-q_{M}\right)^{2}} \text {. }
$$

Substituting in (5) gives Fisher's incorrect formula (2). If, however, we remember that we can only use equation (8) when $q_{M}=1$, then the solution of $(8)$ is

$$
q_{A}=\mathrm{I}-\sqrt{u / s}
$$

which has been given previously. Thus if the requirement $i \rightarrow \infty$ as $q_{M} \rightarrow \mathrm{I}$ is really an " essential point " of Fisher's theory, then the theory is no longer tenable.

On the other hand Fisher may be wrong in saying that this is an essential point; this question is discussed later when the various factors concerning the evolution of dominance are considered. 


\section{A SUGGESTION OF PARSONS AND BODMER}

One of the earliest criticisms of Fisher's theory (Wright, 1929a) was that $\Delta q_{M}$ was of the order of the mutation rate $A \rightarrow a$ and was therefore very small. This follows immediately from the corresponding low frequency of the heterozygote $A a$. In order to meet criticisms of this sort, Parsons and Bodmer (I96I) have put forward the suggestion that we should generally consider the case where the frequency of $A$ is initially small, due to a selective advantage of $a$ over $A$. Then due to a changing environment, $A$ develops a selective advantage over $a$ and the frequency of $A$ increases from near zero to near unity. This situation is exemplified by the phenomenon of industrial melanism. During the course of the increase of $q_{A}$ there will be a large number of heterozygotes $A a$ available for the dominance modification process to act. The main problem is to find how much the frequency $q_{M}$ increases as $q_{A}$ increases from near zero to near unity. In this section we examine this problem mathematically.

It will be convenient for us to ignore any mutation during the process. This assumption will cause only a negligible error. Further, we shall generalise the problem by allowing the value of $k$ (assumed above to be zero) to be an arbitrary positive quantity satisfying $0 \leqq k \leqq h \leqq \mathrm{r}$. Doing this, we get

$$
\begin{array}{r}
\Delta q_{A}=s q_{A}\left(\mathrm{I}-q_{A}\right)\left[\mathrm{I}-q_{A}+\left(2 q_{A}-\mathrm{r}\right)\left(\mathrm{I}-q_{M}\right)\left(2 k q_{M}+h-h q_{M}\right)\right] \\
+ \text { small order terms, } \\
\Delta q_{M}=s q_{A}\left(\mathrm{I}-q_{A}\right)\left[4 k q_{M}+2 h-2 h q_{M}-2 k\right] q_{M}\left(\mathrm{I}-q_{M}\right) \\
+ \text { small order terms. }
\end{array}
$$

Hence, ignoring small order terms and passing to continuous time we get

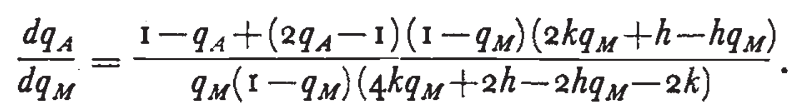

This is a linear differential equation in $q_{A}$ and is therefore soluble immediately. In some cases, however, the solution is given in terms of an integral which cannot be evaluated explicitly, and numerical methods may be required. We consider below some examples of the solution of (9).

Example I.

When $k=0, \frac{1}{2} h=\theta$, solution of (9) yields

$$
\begin{aligned}
q_{A}=\theta^{-\theta} q_{M}^{1-\theta}\left(\mathrm{I}-q_{M}\right)^{\theta} \exp \left\{-\theta /\left(\mathrm{I}-q_{M}\right)\right\} & \\
& \times \int_{K}^{\theta /\left(1-q_{M}\right)}\left(z^{2}-\frac{1}{2} \theta\right)(z-\theta)^{\theta-2} e^{z} d z .
\end{aligned}
$$

This can be evaluated (at any rate simply) only when $\theta=2,3,4, \ldots$, corresponding to $h=\frac{1}{4}, \frac{1}{8}, \frac{1}{8}, \ldots$.

In the case $\theta=2$ we get

$$
q_{A}=\left(\mathrm{I}+q_{M}\right)^{2} / 4 q_{M}-K q_{M}{ }^{-1}\left(\mathrm{I}-q_{M}\right)^{2} \exp \left\{-2 /\left(\mathrm{I}-q_{M}\right)\right\} .
$$

The constant $K$ is fixed by allocating the value of $q_{M}$ when $q_{A}=0$. 
Now in a process such as the one under consideration, we cannot always expect the initial value of $q_{M}$ to be large because when the allele $a$ has a selective advantage over $A$, the dominance-modification behaviour of $M$ will ensure that $M$ is at a selective disadvantage to $m$. In fact if Fisher's own arguments are correct we should take the initial frequency of $M$ to be near zero. If we give the purely arbitrary value of $\frac{1}{2}$ to the initial value of $q_{M}$, then $K=9 e^{4} / 4$ and (10) shows that when $q_{A}$ reaches unity, $q_{M}$ has only increased to 0.677 .

Example 2.

If $k=\frac{1}{2}, h=\mathrm{I}$, then from (9)

$$
q_{A}=q_{M}\left(\mathrm{I}-q_{M}\right) \ln \left\{q_{M} /\left(\mathrm{I}-q_{M}\right)\right\}+q_{M}+K q_{M}\left(\mathrm{I}-q_{M}\right) .
$$

Again putting $q_{M}=\frac{1}{2}$ when $q_{A}=0$ we find $K=-2$ and hence $q_{M}=0.955$ when $q_{A}=\mathrm{I}$.

In table I we summarise the trajectories of $q_{A}$ for various $q_{M}, h$, $k$ and $\pi$ (the initial value of $q_{M}$ ). The values in columns $\mathrm{r}, 2,3$ and 5 were derived by solving (9) explicitly; for columns 4 and 6 the values were obtained by solving (9) numerically.

Two comments may be made about this table. The first is that in only one case does the frequency $q_{M}$ actually reach unity; generally speaking the value of $\pi^{*}$ falls well short of this value. We infer that the process suggested by Parsons and Bodmer for the fixation of $M$ generally cannot be expected to be effective without the help of some further agency, for instance random drift or a selective advantage of $M$ from other causes. We discuss these possibilities in the next section.

The second comment is that Crosby ( 1963 ) has performed two Monte Carlo experiments corresponding to the case $h=\mathrm{I}, k=\frac{1}{2}$, $\pi_{0}=\frac{1}{2}$. He derived the values $\pi^{*}=0.63, \pi^{*}=0.68$ in the two experiments, whereas the above theory suggests that $\pi^{*}=0.96$. The present author felt initially that the value $\pi^{*}=0.96$ may not have been appropriate for the case considered by Crosby, who used $s=\frac{1}{2}$ in his experiments, which may have been too large for the approximations (4) to be valid. Thus an exact analysis was also carried out for this case. The gametic frequencies $c_{1}, c_{2}, c_{3}$ and $c_{4}$ of $A M, a M$, $A m$ and $a m$ were calculated for each generation using the recursive formulæ ( 14 ) to ( 17 ) of Moran ( 1964 ). This procedure is exact and should give an indication of the accuracy of table $I$. The results are summarised in table 2.

After 40 generations the changes in $q_{A}$ and $q_{M}$ become very small and of the order of magnitude of the rounding errors, so that calculations were stopped at that point. However there can be no doubt that the exact values given in table 2 agree very closely with the approximate values in table $\mathrm{I}$, and that the value of $\pi^{*}$ will indeed be very close to $0 \cdot 96$. The reconciliation of Crosby's results with those of table 2 must await further explanation. 


\section{TABLE ?}

Values of $\mathrm{q}_{\mathrm{A}}$ for given values of $\mathrm{q}_{\mathrm{M}}, \mathrm{h}$ and $\mathrm{k}$

$\pi_{0}=$ initial value of $\mathrm{q}_{M}$.

$\pi^{*}=$ value of $\mathrm{q}_{\mathrm{M}}$ when $\mathrm{q}_{\mathrm{A}}=1$.

$\pi_{0}=0.25$

\begin{tabular}{|c|c|c|c|c|c|c|}
\hline$q_{M}$ & $\begin{aligned} h & =t, \\
k & =0\end{aligned}$ & $\begin{array}{c}h=t, \\
k=0\end{array}$ & $\begin{array}{l}h=\frac{1}{t}, \\
k=\frac{1}{8}\end{array}$ & $\begin{aligned} h & =1, \\
k & =0\end{aligned}$ & $\begin{array}{c}h=1 \\
k=\frac{1}{2}\end{array}$ & $\begin{array}{l}h=1, \\
k=:\end{array}$ \\
\hline $\begin{array}{l}0.3 \\
0.35 \\
0.4 \\
0.45 \\
0.5 \\
0.55 \\
0.6 \\
0.65 \\
0.7 \\
0.75 \\
0.8 \\
0.85 \\
0.9\end{array}$ & $\begin{array}{c}0.64 \\
0.90 \\
1.00 \\
\ldots \\
\ldots \\
\ldots \\
\ldots \\
\ldots \\
\ldots \\
\ldots \\
\ldots \\
\ldots \\
\ldots\end{array}$ & $\begin{array}{c}0.4^{8} \\
0.75 \\
0.90 \\
0.99 \\
\ldots \\
\ldots \\
\ldots \\
\ldots \\
\ldots \\
\ldots \\
\ldots \\
\ldots \\
\ldots\end{array}$ & $\begin{array}{c}0.61 \\
0.90 \\
\ldots \\
\ldots \\
\ldots \\
\cdots \\
\ldots \\
\ldots \\
\ldots \\
\cdots \\
\ldots \\
\ldots \\
\ldots\end{array}$ & $\begin{array}{c}0.08 \\
0.17 \\
0.28 \\
0.38 \\
0.48 \\
0.48 \\
0.68 \\
0.78 \\
0.87 \\
0.94 \\
1.00 \\
\ldots \\
\ldots\end{array}$ & $\begin{array}{c}0.07 \\
0.16 \\
0.25 \\
0.35 \\
0.44 \\
0.54 \\
0.64 \\
0.74 \\
0.83 \\
0.91 \\
0.98 \\
\ldots \\
\ldots\end{array}$ & $\begin{array}{l}0.04 \\
0.09 \\
0.15 \\
0.22 \\
0.30 \\
0.38 \\
0.46 \\
0.55 \\
0.63 \\
0.72 \\
0.80 \\
0.89 \\
0.96\end{array}$ \\
\hline \multicolumn{6}{|c|}{$\pi_{0}=0.5$} & 0.93 \\
\hline $\begin{array}{l}0.55 \\
0.6 \\
0.65 \\
0.7 \\
0.75 \\
0.8 \\
0.85 \\
0.9 \\
0.95 \\
1.00\end{array}$ & $\begin{array}{c}0.72 \\
0.95 \\
\ldots \\
\ldots \\
\ldots \\
\ldots \\
\ldots \\
\ldots \\
\ldots \\
\ldots\end{array}$ & $\begin{array}{c}0.56 \\
0.85 \\
0.97 \\
\ldots \\
\ldots \\
\ldots \\
\ldots \\
\ldots \\
\ldots \\
\ldots\end{array}$ & $\begin{array}{c}0.53 \\
0.82 \\
0.97 \\
\ldots \\
\ldots \\
\ldots \\
\ldots \\
\ldots \\
\ldots \\
\ldots\end{array}$ & $\begin{array}{c}0.15 \\
0.31 \\
0.48 \\
0.64 \\
0.78 \\
0.91 \\
0.99 \\
\ldots \\
\ldots \\
\ldots\end{array}$ & $\begin{array}{c}0.10 \\
0.22 \\
0.34 \\
0.46 \\
0.58 \\
0.70 \\
0.82 \\
0.92 \\
0.99 \\
\ldots .\end{array}$ & $\begin{array}{l}0.05 \\
0.11 \\
0.19 \\
0.26 \\
0.35 \\
0.45 \\
0.55 \\
0.66 \\
0.79 \\
0.96\end{array}$ \\
\hline$\pi^{*}$ & 0.64 & 0.68 & 0.67 & 0.86 & 0.96 & 1.00 \\
\hline
\end{tabular}

TABLE 2

Values of $c_{1}, c_{2}, c_{3}, c_{4}$ (frequencies of the gametes AM, aM, Am, am) for various generations in the process $\mathrm{k}=\frac{1}{2}, \mathrm{~h}=\mathrm{I}, \pi_{0}=\frac{1}{2}$

\begin{tabular}{|c|c|c|c|c|c|c|c|}
\hline \multirow{2}{*}{ Generation } & $c_{2}$ & $c_{3}$ & $c_{3}$ & $c_{1}$ & $q_{M}=c_{1}+c_{3}$ & $q_{A}=c_{1}+c_{3}$ & $q_{A} q_{M}$ \\
\hline & 0.005 & 0.495 & 0.005 & 0.495 & 0.500 & 0.010 & 0.005 \\
5 & 0.053 & 0.486 & 0.027 & 0.434 & 0.539 & 0.080 & 0.043 \\
10 & 0.299 & 0.404 & 0.112 & 0.186 & 0.703 & 0.411 & 0.288 \\
15 & 0.608 & 0.225 & 0.130 & 0.038 & 0.832 & 0.738 & 0.614 \\
20 & 0.750 & 0.128 & 0.110 & 0.012 & 0.878 & 0.860 & 0.755 \\
25 & 0.816 & 0.083 & 0.094 & 0.006 & 0.900 & 0.911 & 0.819 \\
30 & 0.853 & 0.059 & 0.084 & 0.004 & 0.912 & 0.937 & 0.855 \\
35 & 0.876 & 0.044 & 0.078 & 0.002 & 0.920 & 0.953 & 0.877 \\
40 & 0.891 & 0.035 & 0.073 & 0.001 & 0.926 & 0.964 & 0.893 \\
\hline
\end{tabular}


It may be noticed that columns 2 and 8 in table 2 show that, except in the early stages of the process, the approximation $c_{1}=q_{A} q_{M}$ used to compute table I is generally correct to within I per cent., even though in this case $s$ is as high as $\frac{1}{2}$.

\section{CONCLUSIONS}

In a finite population, the question of whether the frequency $q_{M}$ will move to unity, so that $A$ becomes dominant over $a$, depends on the factors listed below.

(i) The " initial value" of $q_{M}$;

(ii) The selective advantage of $M$ derived from dominance modification;

(iii) Any other selective advantage or disadvantage of $M$;

(iv) Random sampling.

We now examine the relative importance of these factors in finite populations, but before doing so we consider the deterministic behaviour in the infinite population case. Here we may ignore (i) and (iv), and $\Delta q_{M}$ assumes the form

$$
\Delta q_{M}=C q_{M}\left(\mathrm{I}-q_{M}\right)^{2}+B q_{M}\left(\mathrm{I}-q_{M}\right)
$$

The first term on the right-hand side corresponds to the selective advantage of $M$ through dominance modification and the second term to "other selective pressures". We have seen that when $u=10^{-6}, s=0.01, C$ is at most of order $10^{-4}$; even when $s=\mathrm{I} \cdot 0$, the highest possible value, $C$ is at most of order $10^{-3}$. So far as $B$ is concerned, we may assume it is equally likely to be positive or negative. Whenever $B$ is positive, $\Delta q_{M}$ is positive and $M$ will become fixed. However, when $B$ is negative, $\Delta q_{M}$ will always be negative for $q_{M}$ sufficiently large and $M$ cannot become fixed. (This contrasts with the behaviour using Fisher's incorrect formula, for which $\Delta q_{M}$ can always be made positive for $q_{M}$ large enough, so that $M$ would become fixed.)

Thus when $B$ is negative but less than $A$ in absolute value, $q_{M}$ approaches the value $\mathrm{I}-|B| / C$, which is a point of stable equilibrium. In this case we would expect that in some individuals in the population the allele $A$ would be dominant, in other cases the heterozygote would be intermediate.

When $B$ is negative and greater than $C$ in absolute value, $\Delta q_{M}$ is always negative, $q_{M}$ would approach zero and $A$ would not be dominant.

Since $C$ is generally fairly small, we expect that in 50 per cent. of cases $A$ will be dominant, in a small percentage of cases $A$ would be dominant in some individuals but not in others, and in almost 50 per cent. of cases, $A$ would not be dominant.

Thus it is of some importance to know how the "distribution of other selective advantages $B$ " looks; it has been assumed here that 
this distribution does not have a large concentration in $(-0.00 \mathrm{I}$, $0.001)$. For a discussion of this question the reader is referred to Ford (1964) whose views seem to support the present assumption. The assertion of Fisher (1934) that since $B$ is equally likely to be positive or negative it can in all cases be taken as zero must be rejected.

We turn now to the finite population case. It is well known that if the population size is $\mathcal{N}$ and $|\mathcal{N}(B+C)|>\mathrm{I}$, the behaviour of the population can be taken as deterministic and the above results for an infinite population will apply.

When $|\mathcal{N}(B+C)|<\mathrm{IO}^{-1}$, the dominant factors affecting $q_{M}$ are (i) and (iv). Now $C$ is generally of order $\mathrm{IO}^{-4}$ and is at most of order I $^{-3}$ so that when $\mathcal{N}<$ Iooo, $B=0$, it is possible that the process suggested by Parsons and Bodmer will be the most relevant factor. When $1^{-1}<|\mathcal{N}(B+C)|<\mathrm{Io}$, all factors (i)-(iv) will play some part and we can generally expect that $A$ will be dominant in somewhat more than 50 per cent. of cases.

Other properties of the process have been given in (Ewens, 1965).

\section{SUMMARY}

Corrections are made to a formula of Fisher for the rate of increase in modifiers in the evolution of dominance. The corrections lead to quite different results than those obtained by Fisher. A suggestion of Parsons and Bodmer concerning a method of evolution of dominance is considered mathematically. The importance of this and other factors affecting dominance modification are discussed.

\section{REFERENCES}

CRosBy, J. L. 1963. The evolution and nature of dominance. F. Theoret. Biol., $5,35-5 \mathrm{I}$.

EWENs, W. J. 1965. A note on Fisher's theory of the evolution of dominance. To appear in Ann. Hum. Genet.

FISHER, R. A. 1928a. The possible modification of the response of the wild type to recurrent mutations. Amer. Nat., 62, 1 I 5-1 26.

FISHER, R. A. 1928b. Two further notes on the origin of dominance. Amer. Nat., $62,571-574$.

FISHER, R. A. 1929. The evolution of dominance; reply to Professor Sewall Wright. Amer. Nat., 63, 553-556.

PORD, E. B. I964. Ecological Genetics. Methuen, London and John Wiley, New York.

MORAN, P. A. P. 1964. On the nonexistence of adaptive topographies. Ann. Hum. Genet., 27, 383-393.

PARSONS, P. A., AND BODMER, W. F. 196r. The evolution of overdominance; natural selection and heterozygote advantage. Nature, London, 190, 7-12.

WRIGHT, s. 1929a. Fisher's theory of dominance. Amer. Nat., 63, 274-279.

WRIGHT, s. 1929b. The evolution of dominance. Comment on Dr Fisher's reply. Amer. Nat., 63, 556-561. 